\title{
Human Cytomegalovirus Infection in Nonimmunocompromised Patients: A Retrospective Analysis and Review of the Literature
}

\author{
Konstantin Zedtwitz-Liebenstein ${ }^{a}$ Magda Diab-Elschahaw ${ }^{b}$ Michael Frass ${ }^{c}$

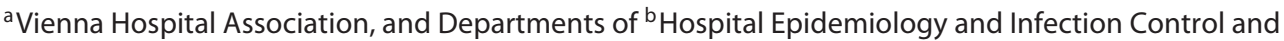 \\ 'Medicine I, Medical University of Vienna, Vienna, Austria
}

\section{Keywords}

Human cytomegalovirus · Fatigue · Immunocompetence

\begin{abstract}
In a retrospective analysis of patients visiting the Outpatient Department of the Division of Infectious Diseases and Tropical Medicine over a period of 3 years, we found a high incidence of symptomatic nonsevere human cytomegalovirus (HCMV) infection in nonimmunocompromised patients. We present 11 symptomatic cases of HCMV infection in nonimmunocompromised patients with non-life-threatening symptoms like long-lasting depletion, fatigue, and subfebrile fever with or without muscular pain and arthralgia. Although the symptoms were not life-threatening, all of the patients suffered a prolonged duration of the disease until improvement. These cases reinforce the important fact that HCMV infections in nonimmunocompromised patients are not always asymptomatic. Therefore, HCMV infection should always be included in the differential diagnosis of patients with unspecific signs of disease like depletion, subfebrile fever, and arthralgia.

(c) 2017 S. Karger AG, Basel
\end{abstract}

\section{Introduction}

Human cytomegalovirus (HCMV) is a betaherpesvirus that infects a large proportion of the human population. The infection is usually asymptomatic in immunocompetent individuals but it can cause severe diseases in immunocompromised patients and pregnant women, inducing congenital infection.

Although HCMV infection is common in immunocompetent hosts, most infections are asymptomatic. The most common clinical presentation is mononucleosislike syndrome (CMV mononucleosis).

Many HCMV infections in immunocompetent adult patients are not primary infections, and they are probably due to reinfection with another strain or reactivation of a latent virus. Laboratory results cannot differentiate between primary and nonprimary infections on many occasions [1].

The high incidence of symptomatic HCMV infections in immunocompetent adults in our Outpatient Department attracted our attention. Severe life-threatening complications of HCMV infection in immunocompetent patients may not be as rare as previously thought [2].

The aim of our study was to evaluate the incidence of symptomatic HCMV infections in immunocompetent

\section{KARGER}

E-Mail karger@karger.com www.karger.com/int
(C) 2017 S. Karger AG, Basel

0300-5526/17/0593-0159\$39.50/0
Konstantin Zedtwitz-Liebenstein

Vienna Hospital Association

Thomas Klestil Platz 7

AT-1030 Vienna (Austria)

E-Mail konstantin.zedtwitz-liebenstein@wienkav.at 
adults. In a systematic review (Cochrane Library) of the period from 1950 to 2007,89 articles reported severe HCMV infections in 290 immunocompetent adults [3]. A case report on children with an atypical presentation of a primary HCMV infection [4] and a review of burns attracted our attention [5].

\section{Methods}

\section{Study Design}

This study was approved by the Ethical Committee of the Medical University of Vienna. In a retrospective analysis of patients visiting the Outpatient Department of the Division of Infectious Diseases and Tropical Medicine over a period of 3 years, we evaluated 32 nonimmunocompromised patients positive for HCMV. The inclusion criteria were: no immunosuppression and no underlying diseases or medication. Twenty-one patients visited the Outpatient Department only once, and clinical follow-up was therefore not available. The 21 excluded patients had mononucleosis-like syndrome (CMV mononucleosis) symptoms and were positive for CMV ELISA IgM antibodies. HCMV polymerase chain reaction (PCR) and further serological examinations were not done. Only 11 patients could be evaluated for this study because the required serological and clinical examinations were documented. The followup for long-lasting clinical symptoms was especially important for our study.

The evaluations performed in our department depended on the investigations done elsewhere and the clinical symptoms. Our diagnostic modalities included serology and qualitative and quantitative PCR for HCMV and Epstein-Barr virus (EBV).

\section{Serological Analysis}

A standard HCMV IgM and IgG ELISA kit (ELISA; Dade Behring $^{\circledR}$, Deerfield, IL, USA) and an ELISA kit for EBV (anti-EBVCA [IgM and IgG], anti-EBNA-1 [IgG], and anti-EBV-EA-D [IgG]; Euroimmun AG, Lübeck, Germany) was used to detect antibodies.

\section{PCR Methods}

A quantitative PCR assay (Cobas Amplicor ${ }^{\circledR}$ CMV Monitor Test; Roche Diagnostics Inc., Branchburg, NJ, USA) amplifying a 365-bp fragment of the HCMV polymerase gene was used for HCMV-DNA detection in plasma. The amplification and detection reactions were expressed as units of HCMV DNA per milliliter of plasma. The assay has a lower limit of sensitivity of 400 HCMV DNA IU/mL [6].

\section{Results}

\section{Patients}

We found a total of 32 patients with HCMV infection without immunosuppression or other underlying diseases or medication. Eleven patients could be included in this study. The median age of the patients ( 4 females and 7 males) included in this study was 38 years (range $28-57$ ).

\section{Clinical Course}

All of the patients reported symptoms like loss of power (long-lasting depletion), fatigue, and fever (subfebrile; Table 1). Some patients reported muscular pain and arthralgia. The laboratory findings in all patients were lymphocytosis (median 49\%, range 43-66, normal range 2540) and elevated lactate dehydrogenase (median $972 \mathrm{U} / \mathrm{L}$, range 386-1,370, normal range 120-240) and liver transaminases (GOT/ASAT median $79 \mathrm{U} / \mathrm{L}$, range 27-156, normal range 0-15; GPT/ALAT median $71 \mathrm{U} / \mathrm{L}$, range 25-98, normal range 0-19; and y-GT median $132 \mathrm{U} / \mathrm{L}$, range 59-174, normal range 4-18) levels. There was no significant elevation of $\mathrm{C}$-reactive protein or other acutephase proteins (fibrinogen). Only the blood sedimentation rate showed an elevation (median $42 \mathrm{~mm} / \mathrm{h}$, range 23-68, normal value 10).

The time until patients were healthy was not exactly evaluable because follow-up after 1 and 2 months showed that symptoms such as fatigue and sickness were still present in all of the patients. Although the laboratory findings normalized (lymphocytosis, lactate dehydrogenase, and liver transaminases), the patients reported persisting fatigue and weakness for many months. The follow-up exam of HCMV IgM was positive in all patients during the period of control.

\section{Evaluations before the Visit to our Outpatient Department}

The approximate time from the beginning of the first symptoms until the diagnosis of an HCMV infection was 20-30 days. All of the patients visited a practitioner first, and one was admitted to another hospital. After an average of 14 days, they visited our Outpatient Department. External evaluations in all of the patients included electrocardiography, serodiagnosis (HCMV not included), pneumomonography, and ultrasound examination (TEE, abdomen), as well as computer tomography (chest, abdomen) in 2 cases and bone marrow puncture in 1 .

\section{Serological Course}

After exclusion of typical viral infections (e.g., EBV), 6 of 11 patients tested positive for HCMV by PCR (median HCMV DNA $120 \mathrm{IU} / \mathrm{mL}$, range 100-1,270, limit of sensitivity 400).

Ten patients were positive for HCMV ELISA IgG and HCMV ELISA IgM in the serological analysis. One patient had seroconversion until a follow-up control evaluation (49 days later; Table 1). 
Table 1. Results of the serological analysis of the 11 patients at the Outpatient Department

\begin{tabular}{|c|c|c|c|c|c|}
\hline $\begin{array}{l}\text { Patient } \\
\text { age, years }\end{array}$ & Sex & $\begin{array}{l}\text { HCMV } \\
\text { IgG }\end{array}$ & $\begin{array}{l}\text { HCMV } \\
\text { IgM }\end{array}$ & $\begin{array}{l}\text { HCMV by } \\
\text { quantitative PCR, } \\
\mathrm{U} / \mathrm{mL}\end{array}$ & Clinical symptoms \\
\hline 55 & $\mathrm{~F}$ & positive & positive & 100 & subfebrile fever, hepatitis, fatigue \\
\hline 34 & $\mathrm{M}$ & positive & positive & negative & muscular pain, fatigue, hepatitis \\
\hline 31 & $\mathrm{M}$ & positive & positive & negative & fatigue, hepatitis \\
\hline 30 & M & positive & positive & 100 & subfebrile fever, arthralgia, hepatitis, fatigue \\
\hline 28 & $\mathrm{~F}$ & negative $^{1}$ & negative $^{1}$ & 619 & subfebrile fever, arthralgia, hepatitis, fatigue \\
\hline 57 & M & positive & positive & 120 & subfebrile fever, hepatitis, fatigue \\
\hline 43 & $\mathrm{M}$ & positive & positive & negative & subfebrile fever, night sweats, hepatitis, fatigue \\
\hline 45 & $\mathrm{~F}$ & positive & positive & negative & subfebrile fever, night sweats, hepatitis, arthralgia, fatigue \\
\hline 37 & $\mathrm{M}$ & positive & positive & 1,270 & muscular pain, hepatitis, arthralgia, fatigue \\
\hline 29 & $\mathrm{M}$ & positive & positive & 317 & subfebrile fever, hepatitis, arthralgia, fatigue \\
\hline 28 & $\mathrm{~F}$ & positive & positive & negative & fatigue, hepatitis \\
\hline
\end{tabular}

HCMV, human cytomegalovirus; PCR, polymerase chain reaction; F, female; $\mathrm{M}$, male.

${ }^{1}$ Seroconversion until a follow-up control evaluation (49 days later).

\section{Discussion}

The diagnosis of a primary HCMV infection in immunocompetent hosts is usually made using serologic studies (either detection of HCMV-specific IgM or a 4 -fold rise in HCMV-specific IgG). These test results provide a presumptive diagnosis in the appropriate clinical setting. The presence of HCMV IgM alone cannot be used to diagnose a primary HCMV infection because IgM can also be present during a secondary HCMV infection, which includes reinfection with a different strain or reactivation of a latent HCMV acquired in the past. IgM-positive results in combination with low IgG avidity (not done in our study) results are considered reliable evidence of a primary infection.

Many HCMV infections in immunocompetent adult patients are not primary infections, and they are probably due to reinfection with another strain or reactivation of a latent virus. These types of infections are very common among adult populations (especially reinfection) and could mimic the symptoms of a primary infection. Laboratory results cannot differentiate between primary and nonprimary infections on many occasions.

According to the paper by Navalpotro et al. [7], not all studies have supported the utility of HCMV PCR assays in the diagnosis of acute HCMV infections in immunocompetent individuals. Using serum obtained from 34 patients with confirmed HCMV mononucleosis, the investigators detected HCMV DNA in the sera of only 7 (21\%) patients using 3 different commercially available assays. The case patients had clinical findings suggestive of acute mononucleosis and laboratory evidence of HCMV seroconversion or HCMV-specific IgM, and diagnostic testing for EBV and human herpesvirus 6 was negative [7].

Although the systematic review (Cochrane Library) of the period from 1950 to 2007 yielded 89 articles reporting severe HCMV infections in 290 immunocompetent adults [3], our patients were clinical different. They all had severe symptoms (i.e., depletion, fatigue, fever [subfebrile], muscular pain, and arthralgia), but they were not critically ill. The main problem was the long-lasting feeling of sickness and depletion, although all laboratory findings normalized.

During our retrospective analysis we found that HCMV infections lasted 20-30 days. All of the patients were relieved to receive a diagnosis of HCMV infection as the explanation for the long disease duration and the slow improvement.

Whether these HCMV infections are a reactivation or maybe a reinfection could not be answered because reactivation is possible in immunocompetent hosts in association with stress and even inflammation or endogenous catecholamines [8].

In our study, a primary infection with HCMV was possible in only 1 patient because of a negative serological analysis (IgM- and IgG-) at beginning of the symptoms. The limitation of our study is that we could not say whether patients positive for IgM and IgG had a primary infection or not, because an HCMV IgG avidity test was miss- 
ing. Although the question of whether each case was a reactivation or a new infection could not be answered, the diagnosis of HCMV in immunocompetent patients has not to be excluded in the differential diagnosis of fever of unknown origin or fatigue.

We could show that HCMV is sometimes an overlooked disease because in a diagnostic evaluation by the practitioner HCMV will not be tested for if the patient is not immunocompromised. Based on our study, we can say that HCMV should be investigated in nonimmunocompromised patients after exclusion of typical viral infections like EBV.

\section{Disclosure Statement}

The authors have no relevant disclosures to make.

\section{References}

1 Shimamura M, Mach M, Britt WJ: Human cytomegalovirus infection elicits a glycoprotein $\mathrm{M}(\mathrm{gM}) / \mathrm{gN}$-specific virus-neutralizing antibody response. J Virol 2006;80:4591-4600.

2 Lancini D, Faddy HM, Flower R, Hogan C: Cytomegalovirus disease in immunocompetent adults. Med J Aust 2014;201:578-580.

3 Rafailidis PI, Mourtzoukou EG, Varbobitis IC, Falagas ME: Severe cytomegalovirus infection in apparently immunocompetent patients: a systematic review. Virol J 2008;5:47.
4 Vigué MG, Tuaillon E, Makinson A, Bullen GM, Foulongne V, Segondy M, Perre PV, Jeziorski E: Lymphoma-like syndrome: 4 case reports about atypical presentation of primary cytomegalovirus infection in immunocompetent children. Medicine 2015;94:1-3.

5 Rennekampff HA, Hamprecht K: Cytomegalovirus infection in burns: a review. J Med Microbiol 2006;55:483-487.

6 Caliendo AM, Schuurman R, Yen-Lieberman B, Spector SA, Andersen J, Manjiry R, Crumpacker C, Lurain NS, Erice A: Comparison of quantitative and qualitative PCR assay for cytomegalovirus DNA in plasma. J Clin Microbiol 2001;39:1334-1338.
7 Navalpotro D, Gimeno C, Navarro D: PCR detection of viral DNA in serum as an ancillary analysis for the diagnosis of acute mononucleosis-like syndrome due to human cytomegalovirus (HCMV) in immunocompetent patients. J Clin Virol 2006;35:193-196.

8 Cook CH, Trgovcich J: Cytomegalovirus reactivation in critically ill immunocompetent hosts: a decade of progress and remaining challenges. Antiviral Res 2011;90:151-159. 